\title{
Local Strain Analysis using Scanning Nanobeam Electron Diffraction during In Situ TEM Nanomechanical Testing
}

Andrew M. Minor ${ }^{1}$

1. Department of Materials Science \& Engineering, University of California, Berkeley, and the National Center for Electron Microscopy, Molecular Foundry, Lawrence Berkeley National Laboratory, Berkeley, CA, USA.

This talk will highlight recent advances with in situ Transmission Electron Microscopy (TEM) nanomechanical testing and imaging techniques that provide insight into small-scale plasticity and the evolution of defect structures in materials. With the advent of fast direct electron detectors, it is possible to perform strain mapping during in-situ deformation in a TEM with the precision of a few nanometers without stopping the experiment (Figure 1) [1]. Our method of local strain mapping consists of recording large multidimensional data sets of nanodiffraction patterns using a high-speed direct electron detectors and custom algorithms for analysis of the collected diffraction pattern datasets [2,3]. These datasets are then reconstructed to form a time-dependent local strain-map with sufficient resolution to measure the transient strains occurring around individual moving dislocations [4]. This talk will describe our recent results from in situ TEM nanomechanical testing that provide insight into multiscale metallurgical phenomena using these techniques, such as the role of short range ordering in Ti alloys, superelastic deformation in oxides and deformation phenomena in metallic glasses [5].

\section{References:}

[1] V. B. Ozdol et al, Applied Physics Letters 106 (2015), p. 253107.

[2] C. Gammer et al, Applied Physics Letters 109 (2016), p. 081906

[3] T. C. Pekin et al, Ultramicroscopy 176 (2017) p. 170

[4] T. C. Pekin et al, Scripta Materialia 146 (2018), p. 87.

[5] Work at the Molecular Foundry is supported by the Office of Science, Office of Basic Energy Sciences, of the U.S. Department of Energy under Contract No. DE-AC02-05CH11231. 


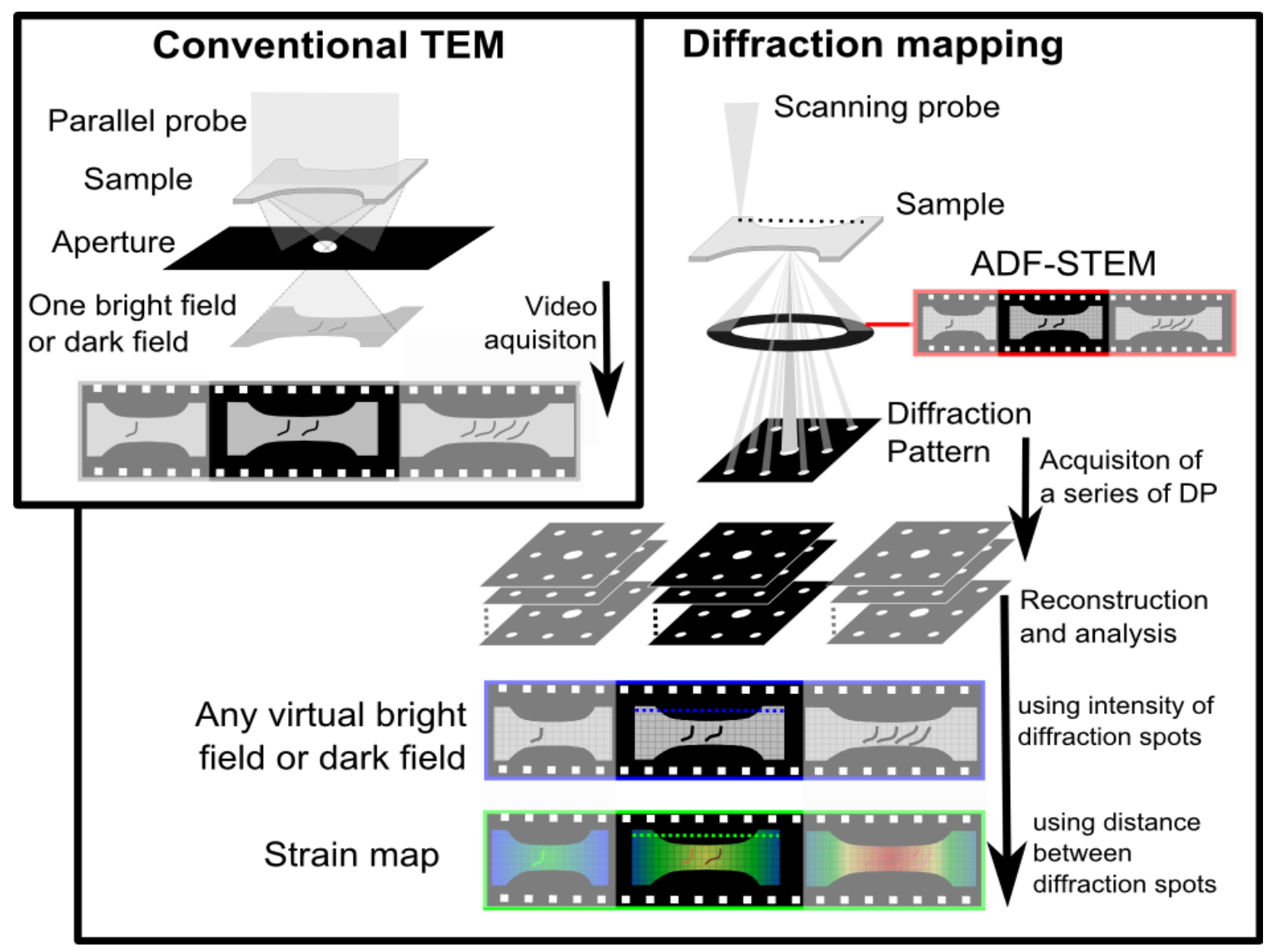

Figure 1. Schematic of scanning nanobeam diffraction maping as applied to in situ tensile deformation 